\title{
VERITAS OBSERVATIONS OF DAY-SCALE FLARING OF M 87 IN 2010 APRIL
}

\author{
E. Aliu ${ }^{1}$, T. Arlen ${ }^{2}$, T. Aune ${ }^{3}$, M. Beilicke ${ }^{4}$, W. Benbow ${ }^{5}$, A. Bouvier $^{3}$, S. M. Bradbury ${ }^{6}$, J. H. Buckley $^{4}$, V. Bugaev $^{4}$,
} K. Byrum ${ }^{7}$, A. Cannon $^{8}$, A. Cesarini ${ }^{9}$, L. Ciupik ${ }^{10}$, E. Collins-Hughes ${ }^{8}$, M. P. Connolly ${ }^{9}$, W. Cui ${ }^{11}$, R. Dickherber $^{4}$, C. Duke ${ }^{12}$, M. Errando ${ }^{1}$, A. Falcone ${ }^{13}$, J. P. Finley ${ }^{11}$, G. Finnegan ${ }^{14}$, L. Fortson ${ }^{15}$, A. Furniss ${ }^{3}$, N. Galante ${ }^{5}$, D. Gall ${ }^{16}$,

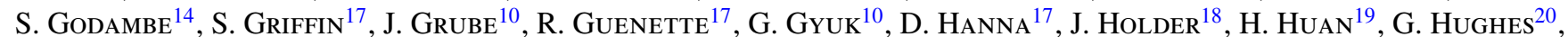
C. M. Hui ${ }^{14}$, T. B. Humensky ${ }^{21}$, A. Imran ${ }^{22}$, P. KaAReT ${ }^{16}$, N. KarlsSON ${ }^{15}$, M. Kertzman ${ }^{23}$, D. KiedA ${ }^{14}$, H. KrawCZYNSKi ${ }^{4}$, F. Krennrich ${ }^{22}$, M. J. Lang ${ }^{9}$, S. LeBohec ${ }^{14}$, A. S Madhavan ${ }^{22}$, G. Maier ${ }^{20}$, P. Majumdar ${ }^{2}$, S. McArthur $^{4}$, A. McCanN $^{17}$, P. Moriarty ${ }^{24}$, R. Mukherjee ${ }^{1}$, P. D. Nuñez ${ }^{14}$, R. A. Ong ${ }^{2}$, M. OrR $^{22}$, A. N. Otte ${ }^{25}$, N. Park $^{19}$, J. S. Perkins $^{26,27}$, A. PiChel $^{28}$, M. Pohl ${ }^{20,29}$, H. Prokoph ${ }^{20}$, J. QuinN ${ }^{8}$, K. Ragan ${ }^{17}$, L. C. Reyes ${ }^{30}$, P. T. Reynolds ${ }^{31}$, E. RoAChE ${ }^{5}$, H. J. Rose ${ }^{6}$, J. Ruppel ${ }^{20,29}$, D. B. Saxon ${ }^{18}$, M. Schroedter ${ }^{5}$, G. H. Sembroski ${ }^{11}$, G. D. Şentürk ${ }^{21}$, C. Skole ${ }^{20}$, D. StaszaK ${ }^{17}$, G. Teší́ ${ }^{17}$, M. Theiling ${ }^{11}$,

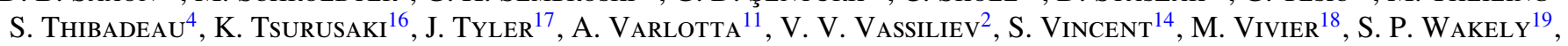
J. E. WARD ${ }^{8}$, T. C. WeEkes ${ }^{5}$, A. Weinstein ${ }^{22}$, T. Weisgarber ${ }^{19}$, D. A. Williams ${ }^{3}$, AND B. Zitzer ${ }^{11}$

${ }^{1}$ Department of Physics and Astronomy, Barnard College, Columbia University, NY 10027, USA

2 Department of Physics and Astronomy, University of California, Los Angeles, CA 90095, USA

${ }^{3}$ Santa Cruz Institute for Particle Physics and Department of Physics, University of California, Santa Cruz, CA 95064, USA

${ }^{4}$ Department of Physics, Washington University, St. Louis, MO 63130, USA

${ }^{5}$ Fred Lawrence Whipple Observatory, Harvard-Smithsonian Center for Astrophysics, Amado, AZ 85645, USA

${ }^{6}$ School of Physics and Astronomy, University of Leeds, Leeds LS2 9JT, UK

${ }^{7}$ Argonne National Laboratory, 9700 S. Cass Avenue, Argonne, IL 60439, USA

${ }^{8}$ School of Physics, University College Dublin, Belfield, Dublin 4, Ireland

${ }^{9}$ School of Physics, National University of Ireland Galway, University Road, Galway, Ireland

${ }^{10}$ Astronomy Department, Adler Planetarium and Astronomy Museum, Chicago, IL 60605, USA

${ }^{11}$ Department of Physics, Purdue University, West Lafayette, IN 47907, USA

${ }^{12}$ Department of Physics, Grinnell College, Grinnell, IA 50112-1690, USA

${ }^{13}$ Department of Astronomy and Astrophysics, 525 Davey Lab, Pennsylvania State University, University Park, PA 16802, USA

${ }^{14}$ Department of Physics and Astronomy, University of Utah, Salt Lake City, UT 84112, USA; cmhui@ physics.utah.edu

${ }^{15}$ School of Physics and Astronomy, University of Minnesota, Minneapolis, MN 55455, USA

${ }^{16}$ Department of Physics and Astronomy, University of Iowa, Van Allen Hall, Iowa City, IA 52242, USA

${ }^{17}$ Physics Department, McGill University, Montreal QC H3A 2T8, Canada

${ }^{18}$ Department of Physics and Astronomy and the Bartol Research Institute, University of Delaware, Newark, DE 19716, USA

${ }^{19}$ Enrico Fermi Institute, University of Chicago, Chicago, IL 60637, USA

${ }^{20}$ DESY, Platanenallee 6, 15738 Zeuthen, Germany

${ }^{21}$ Physics Department, Columbia University, New York, NY 10027, USA

22 Department of Physics and Astronomy, Iowa State University, Ames, IA 50011, USA

${ }^{23}$ Department of Physics and Astronomy, DePauw University, Greencastle, IN 46135-0037, USA

${ }^{24}$ Department of Life and Physical Sciences, Galway-Mayo Institute of Technology, Dublin Road, Galway, Ireland

${ }^{25}$ School of Physics \& Center for Relativistic Astrophysics, Georgia Institute of Technology, 837 State Street NW, Atlanta, GA 30332-0430, USA

${ }^{26}$ CRESST and Astroparticle Physics Laboratory NASA/GSFC, Greenbelt, MD 20771, USA

${ }^{27}$ University of Maryland, Baltimore County, 1000 Hilltop Circle, Baltimore, MD 21250, USA

${ }^{28}$ Instituto de Astronomia y Fisica del Espacio, Casilla de Correo 67-Sucursal 28, (C1428ZAA) Ciudad Autónoma de Buenos Aires, Argentina

${ }^{29}$ Institut für Physik und Astronomie, Universität Potsdam, 14476 Potsdam-Golm, Germany

${ }^{30}$ Physics Department, California Polytechnic State University, San Luis Obispo, CA 94307, USA

${ }^{31}$ Department of Applied Physics and Instrumentation, Cork Institute of Technology, Bishopstown, Cork, Ireland Received 2011 September 16; accepted 2011 December 3; published 2012 February 1

\begin{abstract}
VERITAS has been monitoring the very-high-energy (VHE; > $100 \mathrm{GeV}$ ) gamma-ray activity of the radio galaxy M 87 since 2007. During 2008, flaring activity on a timescale of a few days was observed with a peak flux of $(0.70 \pm 0.16) \times 10^{-11} \mathrm{~cm}^{-2} \mathrm{~s}^{-1}$ at energies above $350 \mathrm{GeV}$. In 2010 April, VERITAS detected a flare from M 87 with peak flux of $(2.71 \pm 0.68) \times 10^{-11} \mathrm{~cm}^{-2} \mathrm{~s}^{-1}$ for $E>350 \mathrm{GeV}$. The source was observed for six consecutive nights during the flare, resulting in a total of $21 \mathrm{hr}$ of good-quality data. The most rapid flux variation occurred on the trailing edge of the flare with an exponential flux decay time of $0.90_{-0.15}^{+0.22}$ days. The shortest detected exponential rise time is three times as long, at $2.87_{-0.99}^{+1.65}$ days. The quality of the data sample is such that spectral analysis can be performed for three periods: rising flux, peak flux, and falling flux. The spectra obtained are consistent with power-law forms. The spectral index at the peak of the flare is equal to $2.19 \pm 0.07$. There is some indication that the spectrum is softer in the falling phase of the flare than the peak phase, with a confidence level corresponding to 3.6 standard deviations. We discuss the implications of these results for the acceleration and cooling rates of VHE electrons in M 87 and the constraints they provide on the physical size of the emitting region.
\end{abstract}

Key words: galaxies: individual (M 87, VER J1230+123) - gamma rays: galaxies

Online-only material: color figures 


\section{INTRODUCTION}

M 87 is a giant radio galaxy located in the Virgo cluster at a distance of $16.7 \mathrm{Mpc}$ (Mei et al. 2007). It is believed to harbor a supermassive black hole of mass $(3.2 \pm 0.9) \times 10^{9} M_{\odot}$ (Macchetto et al. 1997), derived from gas kinematics, or $(6.6 \pm 0.4) \times 10^{9} M_{\odot}$ (Gebhardt et al. 2011), derived from stellar kinematics. Its jet is misaligned with the line of sight; this, along with the proximity of M 87, allows for detailed observations of its structure in the radio (e.g., Cheung et al. 2007), optical (e.g., Biretta et al. 1999), and X-ray (e.g., Marshall et al. 2002; Wilson \& Yang 2002) wavebands. Apparent superluminal motion is observed in the radio and optical wavebands (Biretta et al. 1995, 1999). Month-scale flaring activity has been observed in various energy ranges at the nucleus and at HST-1, the jet feature closest to the nucleus (Perlman et al. 2003; Harris et al. 2009). The jet knot HST-1 is located $0.85 \operatorname{arcsec}(\approx 69 \mathrm{pc}$ projected) from the nucleus and is resolved from the nucleus in the radio, optical, and X-ray energy bands.

Very-high-energy (VHE) gamma-ray emission from M 87 was first detected by HEGRA in 1998/1999 at energies above $730 \mathrm{GeV}$ (Aharonian et al. 2003) and has since been confirmed by H.E.S.S. (Aharonian et al. 2006), VERITAS (Acciari et al. 2008), and MAGIC (Albert et al. 2008). The first gamma-ray flaring activity from M 87 was reported by H.E.S.S. in 2005 , with the flux varying on a timescale of days. The angular resolution of current ground-based gamma-ray instruments is not sufficient to distinguish the different morphological features in M 87, which is therefore detected as a point-like source in VHE gamma rays. However, given the short timescale of the flare, the characteristic size of the gamma-ray emitting region (or of moving regions of low gamma-ray opacity between us and the emitting region) is constrained by the light crossing time of these features and the relativistic Doppler factor of their motion in the observer's reference frame. Under the size constraint, the two most likely regions for gamma-ray production are the unresolved nucleus and the HST-1 knot (Aharonian et al. 2006; Cheung et al. 2007).

During the 2005 gamma-ray flare observed by H.E.S.S., Chandra reported historically maximal flaring from HST-1 (Harris et al. 2006). Through the causality argument, the timescale of the enhanced $\mathrm{TeV}$ emission implies an emission region size of about $R \leqslant 5 \times 10^{15} \delta \mathrm{cm}$, where $\delta$ is the Doppler factor of the radiating region. Aharonian et al. (2006) preferred the nucleus over HST-1 as the VHE gamma-ray production region due to an unrealistically small opening angle $(\sim 1.5 \times$ $10^{-3} \delta \mathrm{deg}$ ) required to channel energy from the central object to the HST-1 knot. However, the Very Long Baseline Array (VLBA) imaged compact knots in HST-1 that are not resolved with semi-minor axes $\leqslant 5 \times 10^{17} \mathrm{~cm}$ (Cheung et al. 2007), and Stawarz et al. (2006) proposed jet reconfinement at the HST-1 location, which can in turn produce $\mathrm{TeV}$ emission. Therefore, HST-1 remains a candidate for TeV emission. However, a VHE gamma-ray flare in 2008 coincided with the historical maximal X-ray flux from the nucleus detected by Chandra, while HST-1 remained in a low state at that time and its X-ray flux was below that of the nucleus. In addition, increasing radio flux from the nucleus, but not from the jet, was observed by the VLBA, lasting up to two months past the VHE gamma-ray flare (Acciari et al. 2009). The 2008 observations therefore favor the nucleus as the origin of the VHE gamma-ray emission.

After the launch of the Fermi Gamma-ray Space Telescope in the summer of 2008, M 87 was also detected in the MeV-GeV energy range by the Fermi Large Area Telescope (LAT; Abdo et al. 2009). However, no significant flaring activity was detected in 2009 at any wavelength.

M 87 has been monitored every year in VHE gamma rays since 2003 by at least one of the three major atmosphericCherenkov telescope arrays-H.E.S.S., MAGIC, and VERITAS. In 2010, VHE flaring activity up to $20 \%$ of the Crab Nebula flux was detected from M 87 in the span of several days (Ong \& Mariotti 2010), and gamma-ray, X-ray, optical, and radio observations were subsequently triggered. Detailed results from the VERITAS observations are presented in this paper, and the multiwavelength light curve will be presented in a separate publication (Abramowski et al. 2011).

\section{OBSERVATIONS AND ANALYSIS}

VERITAS is an array of four $12 \mathrm{~m}$ diameter imaging atmospheric Cherenkov telescopes located at the Fred Lawrence Whipple Observatory in southern Arizona, $1.3 \mathrm{~km}$ above sea level. The telescopes are situated approximately $100 \mathrm{~m}$ apart, forming a convex quadrilateral. Each telescope is equipped with a camera of 499 photomultiplier tubes (PMTs) arranged in a hexagonal lattice covering a field of view with a diameter of 3.5. The array is sensitive to photons with energy from $\sim 150 \mathrm{GeV}$ to more than $30 \mathrm{TeV}$, with an angular resolution of $\sim 0.1$ and an effective area of $\sim 10^{5} \mathrm{~m}^{2}$ at $1 \mathrm{TeV}$. Further description of the VERITAS observatory and its performance are given in Perkins et al. (2009) and Holder et al. (2006).

M 87 was observed between 2009 December and 2010 May for $53.1 \mathrm{hr}$. Observations were conducted at a range of zenith angles between $19^{\circ}$ and $40^{\circ}$, with low elevation excursions (up to $60^{\circ}$ from zenith) during the nights of April 9 through 11 when episodes of flaring were detected. More than $95 \%$ of the data were taken with the full four-telescope array and the remainder with a three-telescope sub-array. To enable simultaneous estimation of source and background signals, the data were accumulated in "wobble mode" for which the source is offset from the camera center by 0.5 in alternating directions every 20 minutes. The analysis presented in this paper is based on $44.6 \mathrm{hr}$ of live time which satisfied data quality and integrity selection criteria.

The data are analyzed with the algorithm described in Acciari et al. (2010). Atmospheric gamma-ray shower images are first corrected for dispersion in PMT gain and timing using information obtained from nightly laser calibrations (Hanna 2008). Then, an image-cleaning process is applied to select pixels with a signal significantly above the night-sky background level. After cleaning, the images are parameterized (Hillas 1985) and the shower direction is reconstructed using the stereoscopic technique (Hofmann et al. 1999). Events are then selected as gammaray-like if at least three camera images pass selection criteria optimized for a source with $1 \%$ of the Crab Nebula flux. The results reported in this paper have all been confirmed by an independent secondary analysis package described in Daniel (2008).

\section{RESULTS}

During the six-month observation period, M 87 was detected at a level of 25.6 standard deviation $(\sigma)$ above the background, with an average flux of $(5.44 \pm 0.30) \times 10^{-12}$ photon $\mathrm{cm}^{-2} \mathrm{~s}^{-1}$ at energies above $350 \mathrm{GeV}$, equivalent to 5\% of the Crab Nebula flux above $350 \mathrm{GeV}$. The following sub-sections first present the daily light curve obtained over six months of observation, then the April flaring episode light curve binned in 20 minute 


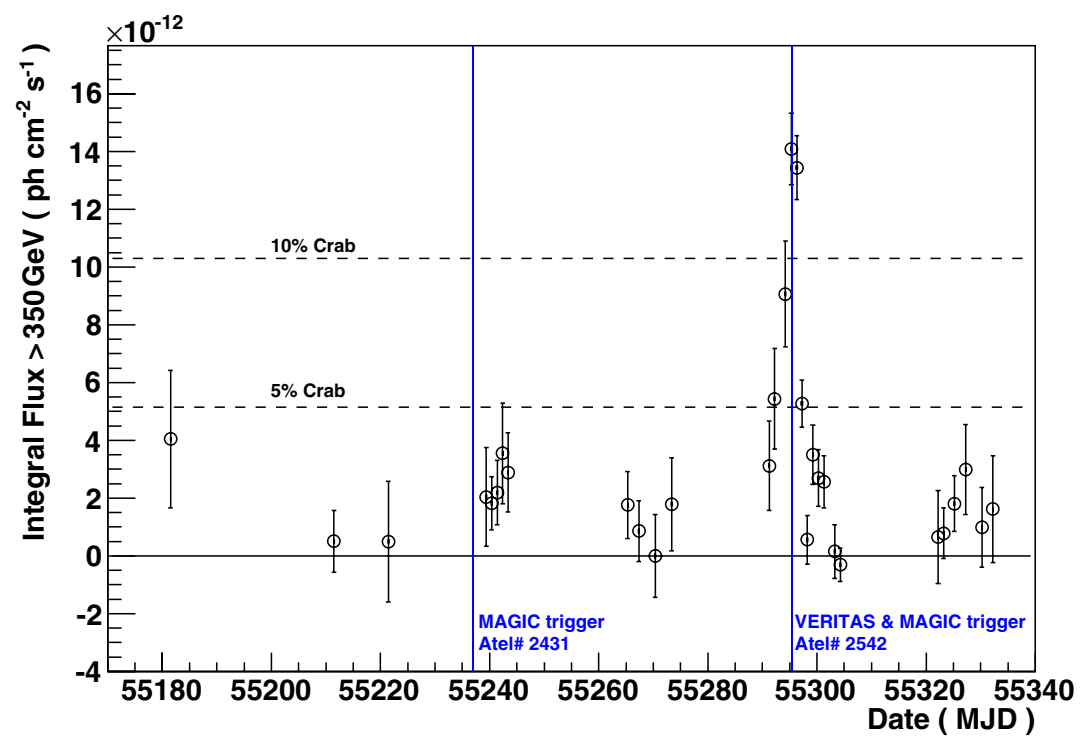

Figure 1. Daily light curve of M 87 observed by VERITAS in 2010. Clear evidence of flaring activity is seen in 2010 April (MJD 55291-55298). Trigger alerts sent by MAGIC on February 10 (MJD 55237) and by VERITAS and MAGIC on April 9 (MJD 55295) are indicated by vertical lines. The average nightly flux during the peak of the flare exceeds $10 \%$ of the Crab Nebula flux at the same energy threshold of $350 \mathrm{GeV}$. A constant spectral index of 2.5 was assumed for the daily flux calculation. (A color version of this figure is available in the online journal.)

intervals, followed by the timescale and spectral analyses of the April flare.

\subsection{Daily Flux Over a Six-month Period}

Figure 1 shows the daily flux recorded by VERITAS between 2009 December and 2010 May. Applying a constant-flux fit to the daily light curve gives a $\chi^{2} /$ dof value of $269.4 / 29$, a strong indication that the flux was not constant during the observation period.

In 2010 February, the MAGIC Collaboration reported an increased activity of M 87 with more than $10 \%$ of the Crab Nebula flux on February 9 (Mariotti 2010). At that time, VERITAS observations were hampered by poor weather conditions, but M 87 was detected by VERITAS in a typical state two nights after the MAGIC alert. In 2010 April, VERITAS detected M 87 with an elevated flux during a week of observations between April 5 and 11 and triggered subsequent multiwavelength observations (Ong \& Mariotti 2010).

\subsection{The 2010 April Flare}

Figure 2 shows the light curve binned in 20 minute intervals during the flare. Observations around the peak of the flare were carried out up to high zenith angles; this reduces the sensitivity at low energies, and as a result, the data points taken at the end of April 10 and the beginning of April 11 have larger uncertainties. The flaring episode began with increasing flux during the nights of April 5 and 6, reaching $10 \%$ of the Crab Nebula flux on April 8. On the following three nights, M 87 was observed for more than five hours each night. The average flux on April 9 and 10 was $15 \%$ of the Crab Nebula flux, reaching as much as $20 \%$ of the Crab Nebula flux in individual 20 minute bins. The average flux on April 11 was 5\% of the Crab Nebula flux. VERITAS continued to monitor M 87 for two hours each night from April 12 to 15, when the flux level returned to a few percent of the Crab Nebula flux, comparable to the low-state flux measured in the past. All flux comparison with the Crab Nebula is at energies above $350 \mathrm{GeV}$.

\subsubsection{Flux Variability Timescale Analysis}

Using the data from April 9 and 10 (MJD 55295 and 55296) when maximal activity occurred, we searched for variability within each night. On April 9, fifteen 20 minute exposures were taken in total and a constant-flux fit yields a $\chi^{2} /$ dof value of $9.3 / 14$ and a corresponding $\chi^{2}$ probability of 0.81 . On April 10, twenty-one 20 minute exposures were taken and the constantflux fit gives a $\chi^{2} /$ dof value of 19.8/20 and a corresponding $\chi^{2}$ probability of 0.47 . In order to investigate variability within a single day of observation in more detail, the wavelet analysis described by Price et al. (2011) is applied to the April 9 and 10 data sets. The highest confidence level for the April 9 data set is obtained for a variability timescale of 80 minutes. However, the confidence level is only $86.2 \%$, implying that on an 80 minute timescale, the evidence for variability is only at the level of $1.5 \sigma$. The highest confidence level for the April 10 data set is obtained for a variability timescale of 160 minutes at $97.5 \%$, or $2.2 \sigma$. Therefore, no evidence for intra-night variability is found.

Figure 3 shows the daily light curve of the April flaring episode. To characterize the timescales of the flare, an exponential function of the form $\Phi=p_{0} e^{(t-55290) / p_{1}}$ is fitted to different periods of the April daily light curve by $\chi^{2}$ minimization. The parameter $p_{1}$ represents the characteristic time of the flux variation. For the days leading up to the flare (MJD 55291-55295, April 5-9), the minimal $\chi^{2} /$ dof value of $0.3 / 2$ is obtained for $p_{1}=2.87$ days. The error bars of the fit parameters $p_{0}$ and $p_{1}$ are determined by finding the parameter ranges with $\chi^{2}$ between $\chi_{\min }^{2}$ and $\chi_{\min }^{2}+2.30$, where $\chi_{\min }^{2}$ is the smallest $\chi^{2}$ value. The same $\chi^{2}$ calculation is repeated for data from the peak flux onward. The details are presented in Table 1. For the period between MJD 55296 and 55304 (April 10-18), the exponential decay time is $1.12_{-0.26}^{+0.31}$ days. An even shorter decay time of $0.90_{-0.15}^{+0.22}$ days is obtained by restricting the fit to the period between MJD 55296 and 55298 (April 10-12). To investigate the possibility of a second flare between MJD 55299 and 55301, a constant-flux fit is applied to data points between MJD 55298 and 55304 (April 12-18). The $\chi^{2} /$ dof value of the 

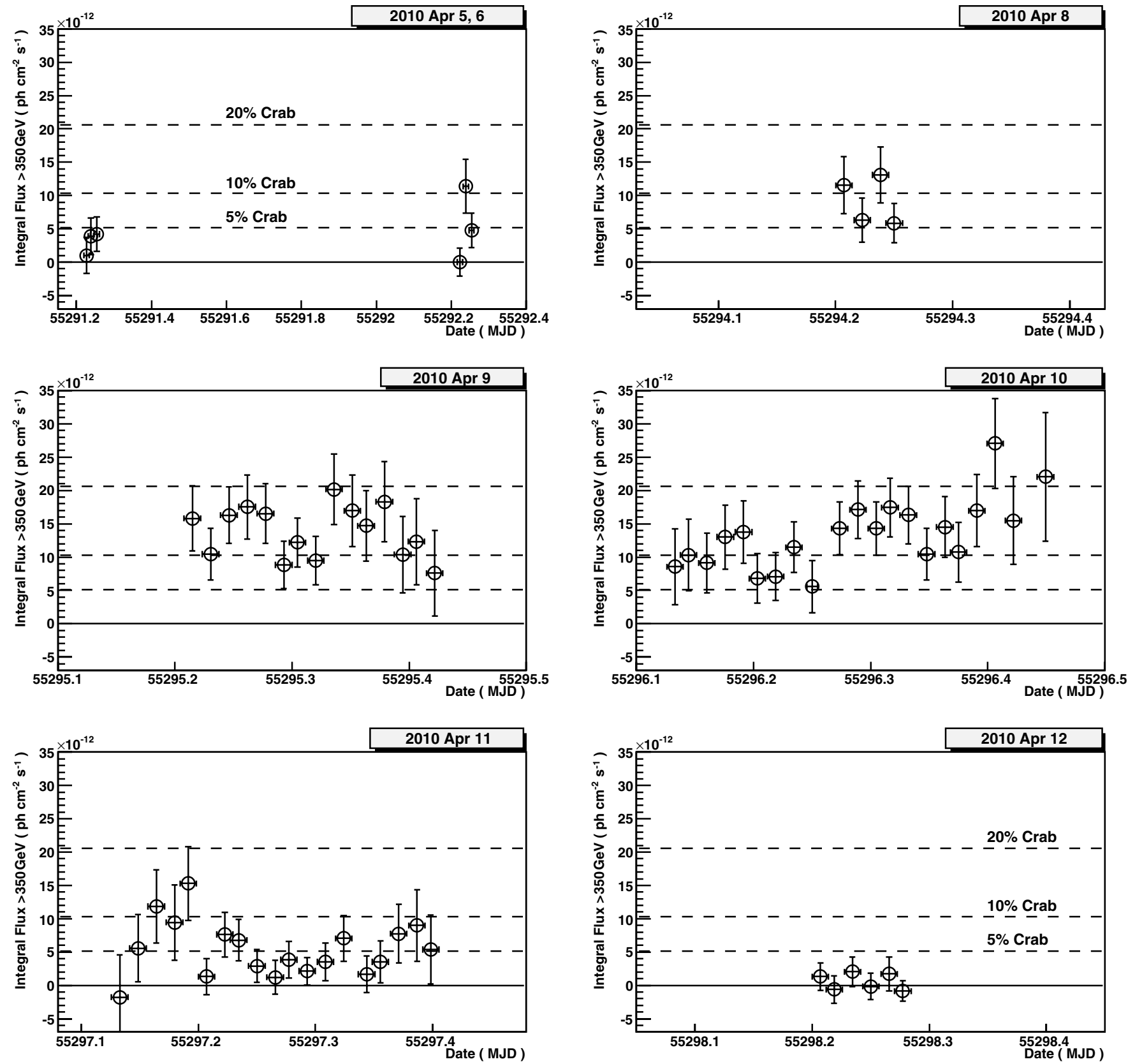

Figure 2. VERITAS light curve with 20 minute binning during the flare period between 2010 April 5 and April 12 (MJD 55291-55298). The flux scale is the same for all six panels, and dashed lines indicating 5\%,10\%, and 20\% of the Crab Nebula flux are included. A constant spectral index of 2.5 was assumed for the flux calculation.

Table 1

$\chi^{2}$ Minimized Parameters of the April Flare Light Curve in Figure 3 (Fit Function $\Phi=p_{0} e^{(t-55290) / p_{1}}$ )

\begin{tabular}{lcccr}
\hline \hline Period (MJD) & $\chi^{2} /$ dof & $\chi^{2}$ Probability & $p_{0}\left(\mathrm{~cm}^{-2} \mathrm{~s}^{-1}\right)$ & $p_{1}$ (days) \\
\hline $55291-55295$ & $0.3 / 2$ & 0.88 & $2.20_{-1.34}^{+1.86} \times 10^{-12}$ & $2.87_{-0.99}^{+1.65}$ \\
$55296-55304$ & $23.7 / 6$ & $6.0 \times 10^{-4}$ & $3.61_{-2.61}^{+17.64} \times 10^{-9}$ & $-\left(1.12_{-0.26}^{+0.31}\right)$ \\
$55296-55298$ & $2.11 / 1$ & 0.15 & $1.48_{-1.32}^{+4.62} \times 10^{-8}$ & $-\left(0.90_{-0.15}^{+0.22}\right)$ \\
\hline
\end{tabular}

Notes. The error bars of $p_{0}$ and $p_{1}$ are statistical only.

constant-flux fit is $9.6 / 4$ with a corresponding $\chi^{2}$ probability of 0.05. In spite of this low confidence level for the constantflux hypothesis, there is nevertheless insufficient evidence to confirm the presence of a second, separated flare component around MJD 55299-55301 (April 13-15).

\subsubsection{Spectral Analysis}

Figure 4 shows the spectra measured during the rising period between April 5 and 8 (MJD 55291-55294), during the peak on April 9 and 10 (MJD 55295-55296), and during the falling period between April 11 and 15 (MJD 55297-55301). 


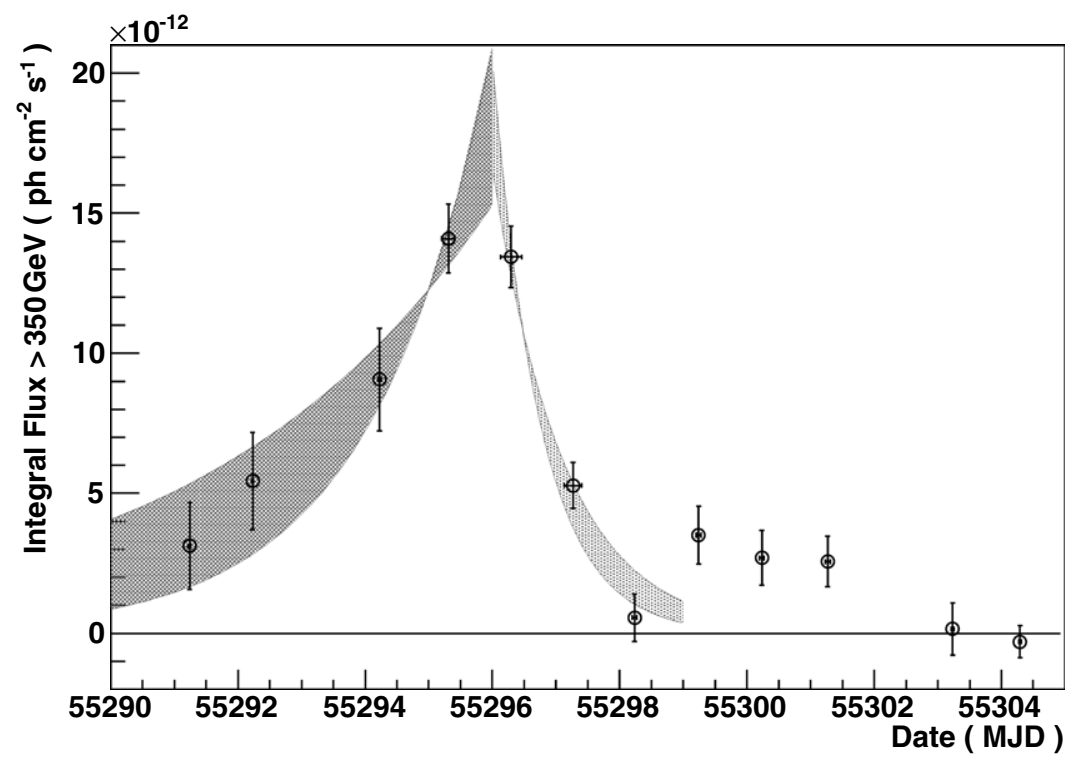

Figure 3. Fits to the 2010 April VHE gamma-ray light curve of M 87 leading up to the flare and trailing the flare, with fit errors included and shown as shaded regions. The exponential timescale is $2.87_{-0.99}^{+1.65}$ days for the rising flux portion, and $0.90_{-0.15}^{+0.22}$ days for MJD 55296-55298 segment of the falling flux.

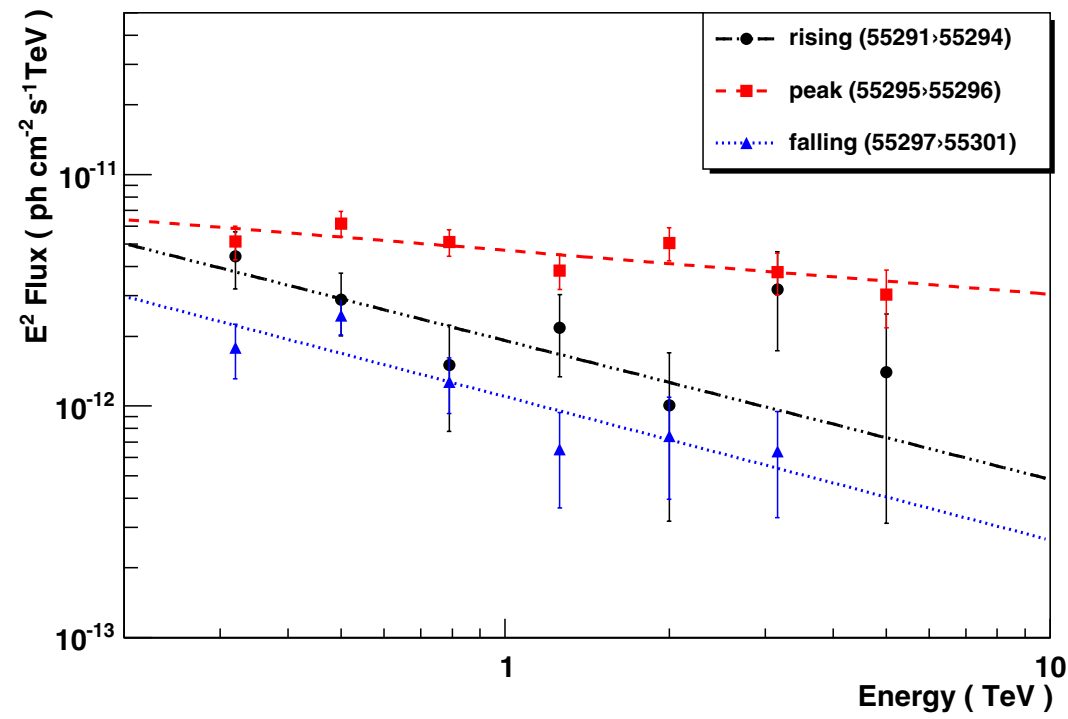

Figure 4. Spectral measurements during three periods: leading up to the flare (MJD 55291-55294, April 5-8), peak of the flare (MJD 55295, 55296, April 9 and 10), and trailing the flare (MJD 55297-55301, April 11-15). The lines are power-law fits to the data, with the values for the flux normalization constant and the spectral index given in Table 2.

(A color version of this figure is available in the online journal.)

Table 2

Spectral Power-law Fit Parameters and Hardness Ratios for the Three Periods of the M 87 Flare in 2010 April: Rising, Peak, and Falling

\begin{tabular}{lccccc}
\hline \hline Periods & MJD Date & $\begin{array}{c}\text { Flux Normalization } \\
\text { Constant } \Phi_{0} \\
\left(0^{-12}\left(\mathrm{~cm}^{-2} \mathrm{~s}^{-1} \mathrm{TeV}^{-1}\right)\right.\end{array}$ & $\begin{array}{c}\text { Spectral Index } \\
\Gamma\end{array}$ & $\chi^{2} /$ dof & Hardness Ratio \\
\hline Rising & $55291-55294$ & $1.92 \pm 0.42$ & $2.60 \pm 0.31$ & $4.1 / 4$ & $0.35 \pm 0.12$ \\
Peak & $55295-55296$ & $4.71 \pm 0.29$ & $2.19 \pm 0.07$ & $4.3 / 5$ & $0.28 \pm 0.03$ \\
Falling & $55297-55301$ & $1.10 \pm 0.16$ & $2.62 \pm 0.18$ & $5.2 / 4$ & $0.10 \pm 0.04$ \\
\hline
\end{tabular}

Notes. Errors given are statistical only.

Power-law fits of the form $\Phi=\Phi_{0}(E / \mathrm{TeV})^{-\Gamma}$ are applied to all three periods, and the corresponding power-law fit parameters are listed in Table 2. The spectral index of the peak period differs from that of the falling period by $2.2 \sigma$, and from that of the rising period by $1.3 \sigma$. The peak period has the hardest spectrum of all three periods.
A hardness ratio (HR) test is also applied to investigate further the possibility of spectral variability between these three different periods. The HR may provide more sensitivity as it is obtained in a straightforward way from the energy distribution of the excess events, whereas the spectral index calculation requires multiple binning and fitting of the data. The HR used 


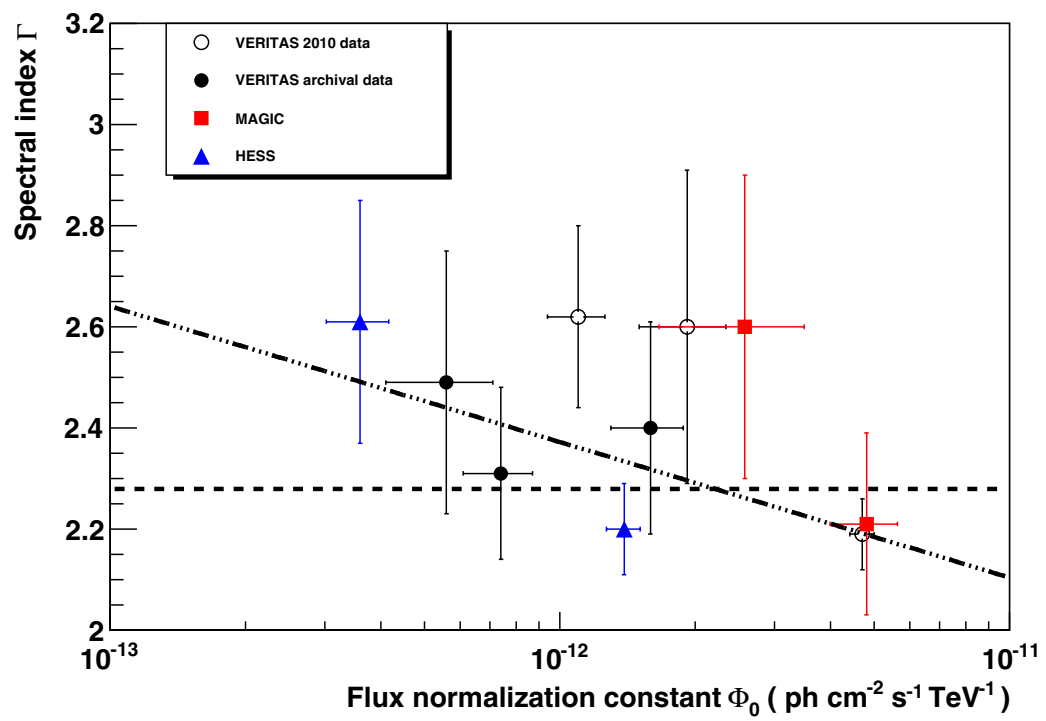

Figure 5. Spectral index vs. flux normalization constant using spectra from the three periods (rising, peak, and falling) and archival spectra from 2004 onward. The dashed line represents a constant fit with a $\chi^{2}$ probability of 0.26 , and the dash-dotted line represents a linear fit of the form $\Gamma=p_{0}+p_{1} \log _{10} \Phi_{0}$ with a $\chi^{2}$ probability of 0.52 . The values of $p_{0}$ and $p_{1}$ are given in the text.

(A color version of this figure is available in the online journal.)

here is defined as the ratio of the integral flux in the energy range $1-10 \mathrm{TeV}$ to that in the range $0.35-1 \mathrm{TeV}$. For the rising period, $\mathrm{HR}=0.35 \pm 0.12$; for the peak period, $\mathrm{HR}=0.28 \pm 0.03$; and for the falling period, HR $=0.10 \pm 0.04$ (see Table 2). The HR for the peak period is found to be larger than that for the falling period with a statistical significance of $3.6 \sigma$, compared to the $2.2 \sigma$ for the spectral index difference of the same time periods. The increased significance may result from a higher sensitivity of the HR to spectral variability. However, we also note that the HR for the rising period is $2.0 \sigma$ larger than that for the falling period while the spectral indices from these periods are identical. This may be a result of statistical fluctuation due to the poor statistics of the rising period spectral measurements.

Figure 5 shows the spectral index $(\Gamma)$ plotted against the flux normalization constant $\left(\Phi_{0}\right)$ for the 2010 April flare spectra (open circles), together with archival VHE gamma-ray spectra from 2004 onward (Aharonian et al. 2006; Acciari et al. 2008, 2009, 2010; Albert et al. 2008). A constant-flux fit to the 2010 April flare flux-index data yields a $\chi^{2}$ probability of 0.05 . A linear fit of the form $\Gamma=p_{0}+p_{1} \log _{10} \Phi_{0}$ yields a $\chi^{2}$ probability of 0.67 , with the parameter $p_{1}=-0.72 \pm 0.30$, which is $2.4 \sigma$ away from zero. Although the fit may suggest a possible correlation between the spectral index and the flux normalization constant, the data do not provide definitive evidence for spectral variability during this flaring episode. Using all the flux-index data available since 2004, a constant-flux fit yields a $\chi^{2}$ probability of 0.26 , while a linear fit yields a $\chi^{2}$ probability of 0.52 with $p_{1}=-0.27 \pm 0.13$.

\section{DISCUSSION}

VERITAS first detected M 87 in 2007 in a low state, with emission at $\sim 2 \%$ of the Crab Nebula flux above $250 \mathrm{GeV}$ (Acciari et al. 2008). In 2008, VERITAS detected flaring activity up to $10 \%$ of the Crab Nebula flux above $250 \mathrm{GeV}$ during a joint monitoring campaign in which correlations between VHE gamma rays, X-ray, and radio (Acciari et al. 2009) were found. In 2009, M 87 was observed to be in a low state again at $\sim 1 \%$ of the Crab Nebula flux above $250 \mathrm{GeV}$ (Acciari et al. 2010). In 2010 April, VERITAS observed the brightest emission ever seen from M 87, with a flux up to $20 \%$ of the Crab Nebula flux above $350 \mathrm{GeV}$. In comparison to previous constraints from past flares (Aharonian et al. 2006; Albert et al. 2008; Acciari et al. 2010), the 2010 VERITAS data set yields the fastest exponential fluxchanging time $\left(0.90_{-0.15}^{+0.22}\right.$ days $)$ ever observed for $M 87$. This time constraint gives a new upper limit on the emission region size that is lower than those derived from previous observations. Using the exponential decay time, the emission region size has a radius of $R \leqslant R_{\mathrm{var}}=\delta c \Delta t=2.3 \times 10^{15} \delta \mathrm{cm} \approx 1.3 \delta R_{s}$, where $R_{s}$ is the Schwarzschild radius of the M 87 black hole $\left(=2 G M_{\mathrm{BH}} / c^{2} \approx 1.8 \times 10^{15} \mathrm{~cm}\right.$, with $M_{\mathrm{BH}}=6.2 \times 10^{9} M_{\odot}$ scaled from Gebhardt et al. (2011) to the distance used in this paper) and $\delta$ is the relativistic Doppler factor. As in earlier findings, this may point to the black hole vicinity as the actual origin of the VHE radiation. While an increased X-ray flux from the nucleus seems to support this hypothesis, no increase of the radio flux from the nucleus could be found (Abramowski et al. 2011), in contrast to the contemporaneous radio and VHE gamma-ray flares observed in 2008 (Acciari et al. 2009).

Another notable characteristic of the 2010 flare is the large difference between the rise time and the decay time of the flux, a feature which has not been seen in previous flares. Since previous VHE flares in 2005 and 2008 were not sampled at a comparable accuracy and their onsets were not as well defined as the 2010 flare, this is the first M87 VHE flare that allows the determination of the rise and fall times. The shape of the 2010 flare also seems less erratic compared to the earlier flares, which could point to a different production mechanism. However, given the lower statistics of the earlier flares, this is difficult to quantify and requires future observations to disentangle.

From a compilation of multiwavelength data sets spanning decades, Wagner et al. (2009) presented a spectral energy distribution (SED) of M 87, along with hadronic and leptonic models. The hadronic synchrotron-proton blazar (SPB) model (Reimer et al. 2004) suggests gamma-ray emission from synchrotron radiation by protons or by muons and pions. However, the SPB model SED produced using archival data before 2004 shows a steep drop-off at $\mathrm{TeV}$ energies that is not compatible with the spectra obtained from the 2010 data set or with 
any previous VHE spectral measurements. Barkov et al. (2010) proposed a scenario where a red giant star, with an envelope of loosely bound material, interacts with the base of the jet. VHE gamma rays are produced near the supermassive black hole via proton-proton interactions between the jet and the red giant cloud. The gamma-ray light curve produced from this model shows an exponential increase/decay time of $\sim 1$ day, identical to the decay timescale obtained from the 2010 data. However, the model gave no prediction on the VHE spectrum for comparison with our data.

There are also several leptonic jet models with different geometric structures that can explain the VHE gamma-ray emission, such as the decelerating jet model by Georganopoulos et al. (2005), the multi-blob synchrotron-self-Compton (SSC) model by Lenain et al. (2008), and the spine-sheath model by Tavecchio \& Ghisellini (2008). The SED solutions obtained from these models can explain the observed rapid variability and match the VHE spectra well in both low and flaring states from 2005 and earlier. Looking into some of these models in more detail, the model parameters can be adjusted to account for recent measurements. In the case of the multi-blob scenario, Lenain et al. (2008) showed that for the case of M 87, the model spectrum hardens with decreasing magnetic field. In order to keep the size of the VHE emitting region of the order of the Schwarzschild radius, the local value of the magnetic field should be $\leqslant 0.01 \mathrm{G}$. The spine-sheath model, however, seems to face difficulties in achieving a harder spectrum due to absorption of $\mathrm{TeV}$ photons from interactions with the optical-IR photons from the spine. As pointed out by Tavecchio \& Ghisellini (2008), severe gamma-ray photon absorption can be alleviated by increasing the emission region size, which would decrease the absorption optical depth. However, this would be limited by the observed short-term variability. Abdo et al. (2009) fitted a homogeneous one-zone SSC model using 2009 VLBA radio, Chandra X-ray, and Fermi-LAT measurements when M 87 was observed to be in a low state from radio to VHE gamma rays. A contemporaneous spectral measurement in the VHE range was not possible due to low statistical significance (Acciari et al. 2010), but compared to archival lowstate VHE measurements, the one-zone SSC model seems to underestimate the VHE gamma-ray flux by almost an order of magnitude. Georganopoulos et al. (2005) and Lenain et al. (2008) demonstrated that one-zone homogeneous models are unlikely to reproduce the observed VHE spectrum.

Giannios et al. (2010) presented a scenario where minijets are formed within the jet due to flow instabilities. These minijets move relativistically with respect to the main jet flow. VHE gamma rays are produced from the interactions between the minijets and the jet, and are beamed with large Doppler factor when the minijets are aligned with our line of sight. The minijets model SED is compatible with the 2010 data. A satisfactory solution for the high state observed by VERITAS in 2010 is also possible within the magnetosphere model (e.g., Neronov \& Aharonian 2007; Rieger \& Aharonian 2008; Vincent \& Lebohec 2010; Levinson \& Rieger 2011). The magnetosphere model is dependent on the injected plasma, which suggests that a vacuum gap with a large electric field that is capable of accelerating electrons to very high energies may be formed during a period of low accretion rate.

We cannot discriminate between different leptonic models based on this VHE data alone. The spectral change with flux level would serve as an important input for the modeling once it is confirmed by a second flare. Leptonic models tend to predict a more direct correlation between X-ray and VHE gamma rays. For the 2010 flare of M 87, extensive follow-up observations of the VHE gamma-ray flare (Ong \& Mariotti 2010) were carried out in X-ray, optical, and radio wavebands. The result is a much more complete sampling across different energy bands than in the case of previous M 87 flares, providing a data set that will help to constrain the emission region and the radiative processes involved. A separate, upcoming publication (Abramowski et al. 2011) will present the multiwavelength result, which spans 16 decades of energy.

This research is supported by grants from the U.S. Department of Energy Office of Science, the U.S. National Science Foundation and the Smithsonian Institution, by NSERC in Canada, by Science Foundation Ireland (SFI 10/RFP/AST2748), and by STFC in the UK. We acknowledge the excellent work of the technical support staff at the Fred Lawrence Whipple Observatory and at the collaborating institutions in the construction and operation of the instrument.

Facility: VERITAS

\section{REFERENCES}

Abdo, A. A., Ackermann, M., Ajello, M., et al. 2009, ApJ, 707, 55

Abramowski, A., Acero, F., Aharonian, F., et al. 2011, ApJ, in press (arXiv:1111.5341)

Acciari, V. A., Aliu, E., Arlen, T., et al. 2009, Science, 325, 444

Acciari, V. A., Aliu, E., Arlen, T., et al. 2010, ApJ, 716, 819

Acciari, V. A., Beilicke, M., Blaylock, G., et al. 2008, ApJ, 679, 397

Aharonian, F., Akhperjanian, A., Beilicke, M., et al. 2003, A\&A, 403, L1

Aharonian, F., Akhperjanian, A. G., Bazer-Bachi, A. R., et al. 2006, Science, 314,1424

Albert, J., Aliu, E., Anderhub, H., et al. 2008, ApJ, 685, L23

Barkov, M. V., Aharonian, F. A., \& Bosch-Ramon, V. 2010, ApJ, 724, 1517

Biretta, J. A., Sparks, W. B., \& Macchetto, F. 1999, ApJ, 520, 621

Biretta, J. A., Zhou, F., \& Owen, F. N. 1995, ApJ, 447, 582

Cheung, C. C., Harris, D. E., \& Stawarz, Ł. 2007, ApJ, 663, L65

Daniel, M. K. 2008, Proc. 30th ICRC, ed. K. Caballero et al. (Vol. 3; Mexico City: Univ. Nacional Autonoma de México), 1325

Gebhardt, K., Adams, J., Richstone, D., et al. 2011, ApJ, 729, 119

Georganopoulos, M., Perlman, E. S., \& Kazanas, D. 2005, ApJ, 634, L33

Giannios, D., Uzdensky, D. A., \& Begelman, M. C. 2010, MNRAS, 402, 1649

Hanna, D. 2008, in Proc. 30th ICRC, ed. K. Caballero et al. (Vol. 3; Mexico City: Univ. Nacional Autonoma de México), 1417

Harris, D. E., Cheung, C. C., Biretta, J. A., et al. 2006, ApJ, 640, 211

Harris, D. E., Cheung, C. C., Stawarz, Ł., Biretta, J. A., \& Perlman, E. S. 2009, ApJ, 699, 305

Hillas, A. M. 1985, in Proc. 19th ICRC (Vol. 3; Washington, DC: NASA)

Hofmann, W., Jung, I., Konopelko, A., et al. 1999, Astropart. Phys., 12, 135

Holder, J., Atkins, R. W., Badran, H. M., et al. 2006, Astropart. Phys., 25, 391

Lenain, J., Boisson, C., Sol, H., \& Katarzyński, K. 2008, A\&A, 478, 111

Levinson, A., \& Rieger, F. 2011, ApJ, 730, 123

Macchetto, F., Marconi, A., Axon, D. J., et al. 1997, ApJ, 489, 579

Mariotti, M. 2010, ATel, 2431, 1

Marshall, H. L., Miller, B. P., Davis, D. S., et al. 2002, ApJ, 564, 683

Mei, S., Blakeslee, J. P., Côté, P., et al. 2007, ApJ, 655, 144

Neronov, A., \& Aharonian, F. A. 2007, ApJ, 671, 85

Ong, R. A., \& Mariotti, M. 2010, ATel, 2542, 1

Perkins, J. S., \& Maier, G. The VERITAS Collaboration. 2009, in Proc. Fermi Symposium eConf, CO91122 (arXiv:0912.3841)

Perlman, E. S., Harris, D. E., Biretta, J. A., Sparks, W. B., \& Macchetto, F. D. 2003, ApJ, 599, L65

Price, R. G., Vincent, S., \& LeBohec, S. L. 2011, Astropart. Phys., 34, 871

Reimer, A., Protheroe, R. J., \& Donea, A. 2004, A\&A, 419, 89

Rieger, F. M., \& Aharonian, F. A. 2008, A\&A, 479, L5

Stawarz, Ł., Aharonian, F., Kataoka, J., et al. 2006, MNRAS, 370, 981

Tavecchio, F., \& Ghisellini, G. 2008, MNRAS, 385, L98

Vincent, S., \& Lebohec, S. 2010, MNRAS, 409, 1183

Wagner, R. M., Beilicke, M., Davies, F., et al. 2009, in Proc. Fermi Symposium eConf, CO91122 (arXiv:0912.3597)

Wilson, A. S., \& Yang, Y. 2002, ApJ, 568, 133 